KYUNGPOOK Math. J. 57(2017), 163-185

https://doi.org/10.5666/KMJ.2017.57.1.163

pISSN 1225-6951 eISSN 0454-8124

(C) Kyungpook Mathematical Journal

\title{
Separating a Chart
}

\author{
Teruo Nagase and Akiko Shima* \\ Department of Mathematics, Tokai University 4-1-1 Kitakaname, Hiratuka Kana- \\ gawa, 259-1292 Japan \\ e-mail: nagase@keyaki.cc.u-tokai.ac.jp andshima@keyaki.cc.u-tokai.ac.jp
}

ABstract. In this paper, we shall show a condition for that a chart is C-move equivalent to the product of two charts, the union of two charts $\Gamma^{*}$ and $\Gamma^{* *}$ which are contained in disks $D^{*}$ and $D^{* *}$ with $D^{*} \cap D^{* *}=\emptyset$.

\section{Introduction}

Charts are oriented labeled graphs in a disk which represent surface braids (see [1], [7], and see Section 2 for the precise definition of charts, see [7, Chapter 14] for the definition of surface braids). In a chart there are three kinds of vertices; white vertices, crossings and black vertices. A C-move is a local modification of charts in a disk. The closures of surface braids are embedded closed oriented surfaces in 4 -space $\mathbb{R}^{4}$ (see [7, Chapter 23] for the definition of the closures of surface braids). A C-move between two charts induces an ambient isotopy between the closures of the corresponding two surface braids. In this paper, we shall show a condition for that a chart is C-move equivalent to the product of two charts (Theorem 1).

We will work in the PL category or smooth category. All submanifolds are assumed to be locally flat. In [4] and [15], we investigated minimal charts with exactly four white vertices. In [16], we showed that there is no minimal chart with exactly five vertices. Hasegawa proved that there exists a minimal chart with exactly six white vertices which represents the surface braid whose closure is ambient isotopic to a 2-twist spun trefoil [3]. In [8] and [14], we investigated minimal charts with exactly six white vertices. We show that there is no minimal chart with exactly seven vertices $([9],[10],[11],[12],[13])$. Thus the next targets are minimal

* Corresponding Author.

Received March 27, 2016; revised November 8, 2016; accepted November 17, 2016.

2010 Mathematics Subject Classification: 57Q45, 57Q35.

Key words and phrases: 2-knot, chart, white vertex.

The second author was supported by JSPS KAKENHI Grant Number 23540107. 


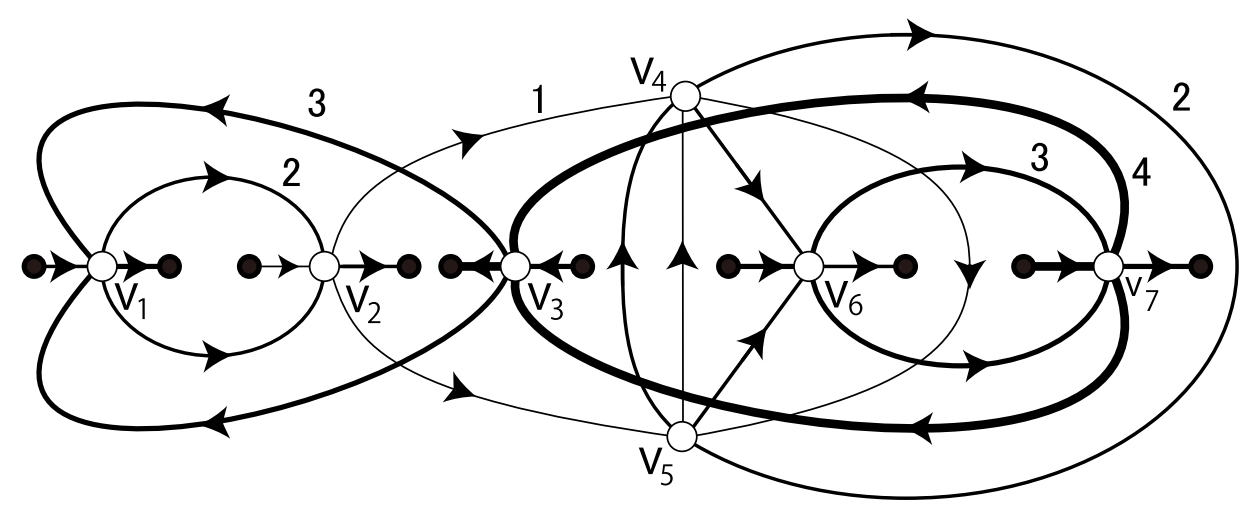

Figure 1: Chart of type $(1 ; 3,2,2)$.

charts with eight or nine white vertices. As an application of Theorem 1, we shall show that there are 12 kinds of types for a minimal chart with eight white vertices (Corollary 2 and Corollary 3), and there are 15 kinds of types for a minimal chart with nine white vertices (Corollary 5).

Let $\Gamma$ be a chart. For each label $m$, we denote by $\Gamma_{m}$ the 'subgraph' of $\Gamma$ consisting of all the edges of label $m$ and their vertices.

Two charts are said to be $C$-move equivalent if there exists a finite sequence of $\mathrm{C}$-moves which modifies one of the two charts to the other.

Now we define a type of a chart: Let $\Gamma$ be a chart, $m$ a label of $\Gamma$, and $n_{1}, n_{2}, \ldots, n_{p}$ integers. The chart $\Gamma$ is said to be of type $\left(m ; n_{1}, n_{2}, \ldots, n_{p}\right)$, or of type $\left(n_{1}, n_{2}, \ldots, n_{p}\right)$ briefly, if it satisfies the following three conditions:

(i) For each $i=1,2, \ldots, p$, the chart $\Gamma$ contains exactly $n_{i}$ white vertices in $\Gamma_{m+i-1} \cap \Gamma_{m+i}$.

(ii) If $i<0$ or $i>p$, then $\Gamma_{m+i}$ does not contain any white vertices.

(iii) Each of the two subgraphs $\Gamma_{m}$ and $\Gamma_{m+p}$ contains at least one white vertex.

Note that $n_{1} \geq 1$ and $n_{p} \geq 1$ by Condition (iii).

The chart $\Gamma$ shown in Figure 1 contains exactly seven white vertices, and $\Gamma_{1} \cap$ $\Gamma_{2}=\left\{v_{2}, v_{4}, v_{5}\right\}, \Gamma_{2} \cap \Gamma_{3}=\left\{v_{1}, v_{6}\right\}$ and $\Gamma_{3} \cap \Gamma_{4}=\left\{v_{3}, v_{7}\right\}$. Hence this chart is a chart of type $(1 ; 3,2,2)$. Note that this chart is not a minimal chart.

Two C-move equivalent charts $\Gamma$ and $\Gamma^{\prime}$ are said to be same $C$-type provided that the types of the two charts are same.

For a subset $X$ of a chart, let

$$
w(X)=\text { the number of white vertices of the chart contained in } X \text {. }
$$

A chart $\Gamma$ is zero at label $k$ provided that 
(i) $\Gamma_{k} \cap \Gamma_{k+1}=\emptyset$,

(ii) there exists a label $i$ with $i \leq k$ and $w\left(\Gamma_{i}\right) \neq 0$, and

(iii) there exists a label $j$ with $k<j$ and $w\left(\Gamma_{j}\right) \neq 0$.

Let $\Gamma$ be an $n$-chart, and $D_{1}, D_{2}$ disjoint disks with $D_{i} \cap \Gamma \neq \emptyset$ for $i=1,2$, $\partial D_{i} \cap \Gamma=\emptyset$ for $i=1,2$, and $D_{1} \cup D_{2} \supset \Gamma$. Then we can consider $\Gamma^{*}=D_{1} \cap \Gamma$ and $\Gamma^{* *}=D_{2} \cap \Gamma$ as $n$-charts. Then we call the chart $\Gamma$ the product of the two charts $\Gamma^{*}$ and $\Gamma^{* *}[6]$.

A chart $\Gamma$ is separable at label $k$ if there exist subcharts $\Gamma^{*}, \Gamma^{* *}$ such that

(i) $\Gamma$ is the product of the two charts $\Gamma^{*}$ and $\Gamma^{* *}$,

(ii) $w\left(\Gamma^{*}\right) \neq 0$ and $w\left(\Gamma^{* *}\right) \neq 0$,

(iii) $w\left(\Gamma_{i}^{*}\right)=0$ for all label $i$ with $k<i$, and

(iv) $w\left(\Gamma_{i}^{* *}\right)=0$ for all label $i$ with $i \leq k$.

The following is our main theorem:

Theorem 1. A chart $\Gamma$ is zero at label $k$ if and only if there exists a chart $\Gamma^{\prime}$ with the same $C$-type of $\Gamma$ such that $\Gamma^{\prime}$ is separable at label $k$.

A chart $\Gamma$ is minimal if it possesses the smallest number of white vertices among the charts C-move equivalent to the chart $\Gamma$ (cf. [5]).

Corollary 2. Let $\Gamma$ be a minimal chart with $w(\Gamma)=8$. If $\Gamma$ is zero at some label, then $\Gamma$ is $C$-move equivalent to one of the following charts:

(a) the product of two charts of type (4).

(b) the product of two charts of type $(2,2)$.

(c) the product of a chart of type (4) and a chart of type $(2,2)$.

The chart shown in Figure 2 contains exactly eight white vertices of type $(2 ; 4,0,2,2)$. This chart is a product of a chart of type a chart of type $(2 ; 4)$ and a chart of type $(4 ; 2,2)$.

Corollary 3. Let $\Gamma$ be a minimal $n$-chart with $w(\Gamma)=8$ such that $\Gamma$ is not zero at any label. If necessary we change all the edges of label $k$ to ones of label $n-k$ for each $k=1,2, \cdots, n-1$ simultaneously, then the type of $\Gamma$ is $(8),(6,2),(5,3)$, $(4,4),(4,2,2),(3,3,2),(3,2,3),(2,4,2)$ or $(2,2,2,2)$.

The chart shown in Figure 3 is a chart of type $(1 ; 2,4,2)$, and represents a 3 -twist spun trefoil [2].

Corollary 4. If $\Gamma$ is a minimal chart with $w(\Gamma)=9$ or 11 , then the chart is not zero at any label. Namely if the type of the chart is $\left(m ; n_{1}, n_{2}, \cdots, n_{p}\right)$, then for each $i=1,2, \cdots, p$, we have $n_{i} \neq 0$. 

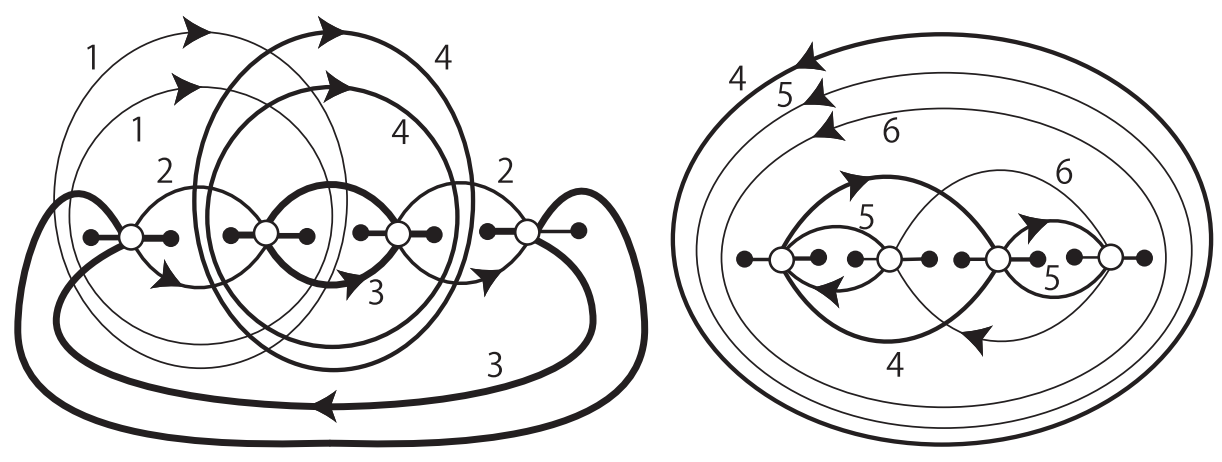

Figure 2: Product of a chart of type $(2 ; 4)$ and a chart of type $(4 ; 2,2)$.

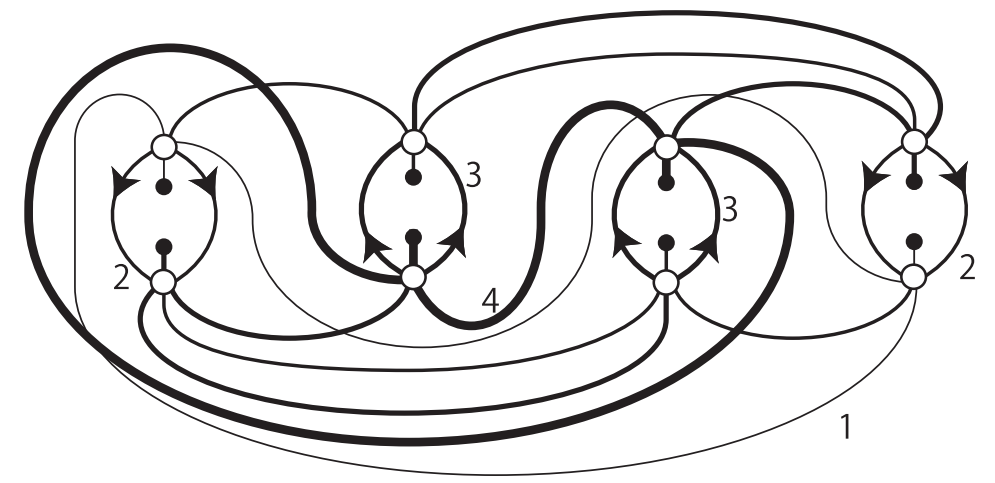

Figure 3: Chart of type $(1 ; 2,4,2)$.

Corollary 5. Let $\Gamma$ be a minimal $n$-chart with $w(\Gamma)=9$. If necessary we change all the edges of label $k$ to ones of label $n-k$ for each $k=1,2, \cdots, n-1$ simultaneously, then the type of $\Gamma$ is $(9),(7,2),(6,3),(5,4),(5,2,2),(4,3,2),(4,2,3),(4,1,4)$, $(3,4,2),(3,3,3),(2,5,2),(4,1,2,2),(3,2,2,2),(2,3,2,2)$ or $(2,2,1,2,2)$.

The paper is organized as follows. In Section 2, we define charts. In Section 3 and Section 4, we prove lemmata that are needed in order to prove Theorem 1. In Section 5 , we define $\omega_{k}$-minimal charts. By using $\omega_{k}$-minimal charts, we show that if a chart $\Gamma$ is zero at label $k$, then there exists a chart $\Gamma^{*}$ obtained from $\Gamma$ by C-I-R2 moves and C-I-M2 moves such that $\Gamma_{k}^{*} \supset \Gamma_{k}$ and all of black vertices and white vertices in $\bigcup_{i=k+1}^{\infty} \Gamma_{i}^{*}$ are contained in the same complementary domain of $\Gamma_{k}$. In Section 6, we give a proof of Theorem 1 and proofs of corollaries. 
(a)

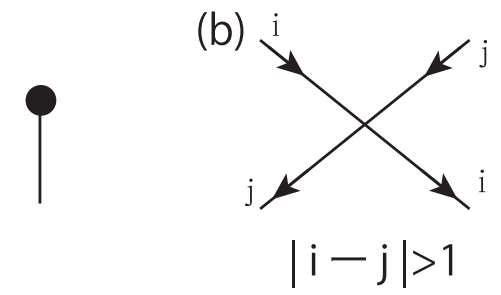

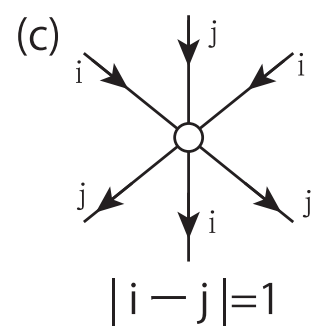

Figure 4: (a) A black vertex. (b) A crossing. (c) A white vertex.

\section{Preliminaries}

Let $n$ be a positive integer. An $n$-chart is an oriented labeled graph in a disk, which may be empty or have closed edges without vertices, called hoops, satisfying the following four conditions:

(i) Every vertex has degree 1, 4, or 6.

(ii) The labels of edges are in $\{1,2, \ldots, n-1\}$.

(iii) In a small neighborhood of each vertex of degree 6 , there are six short arcs, three consecutive arcs are oriented inward and the other three are outward, and these six are labeled $i$ and $i+1$ alternately for some $i$, where the orientation and the label of each arc are inherited from the edge containing the arc.

(iv) For each vertex of degree 4, diagonal edges have the same label and are oriented coherently, and the labels $i$ and $j$ of the diagonals satisfy $|i-j|>1$.

We call a vertex of degree 1 a black vertex, a vertex of degree 4 a crossing, and a vertex of degree 6 a white vertex respectively (see Figure 4).

Let $D_{1}^{2}, D_{2}^{2}$ be disks, and $p r_{2}: D_{1}^{2} \times D_{2}^{2} \rightarrow D_{2}^{2}$ the projection defined by $p r_{2}(x, y)=y$. Let $Q_{n}$ be a set of $n$ interior points of $D_{1}^{2}$. A surface braid $S$ is an oriented surface embedded properly in $D_{1}^{2} \times D_{2}^{2}$ such that the map $\left.p r_{2}\right|_{S}: S \rightarrow D_{2}^{2}$ is a branched covering of degree $n$ and $\partial S=Q_{n} \times \partial D_{2}^{2}$ [7, Chapter 14]. A surface braid can be represented by a motion picture method, a one-parameter family of geometric $n$-braids $\left\{b_{t}\right\}_{t \in[0,1]}$ except for a finite number of values $t_{1}, t_{2}, \cdots, t_{m} \in[0,1]$. A motion picture for a white vertex is a motion picture as shown in Figure 5(a) (cf. [7, p. 132, Figure 18.5]). A motion picture for a crossing is a motion picture as shown in Figure 5(b) (cf. [7, p. 131, Figure 18.4]). A motion picture for a black vertex is a motion picture as shown in Figure 5(c) (cf. [7, p. 134, Figure 18.7]). A black vertex is corresponding to a singular point of a branched covering map.

Now $C$-moves are local modifications of charts in a disk as shown in Figure 6 (cf. [1], [7], [17]). We do not use a C-I-M4 move (a tetrahedral move), and we do 
(a) i $\quad$ i $+1 \quad i+2$
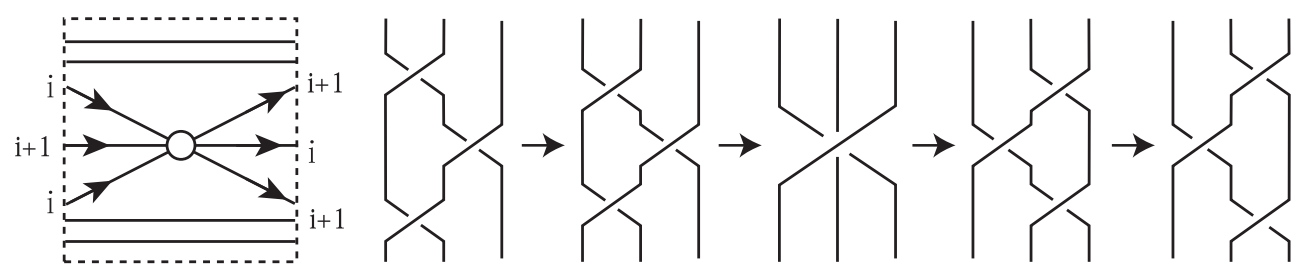

(b)
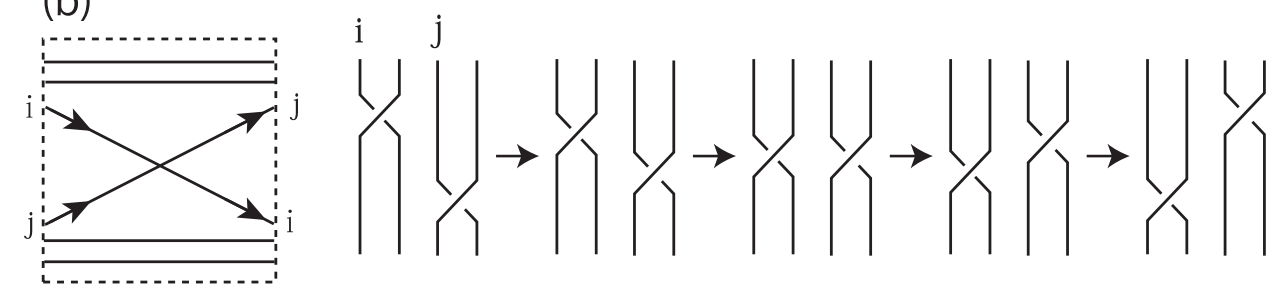

(c)
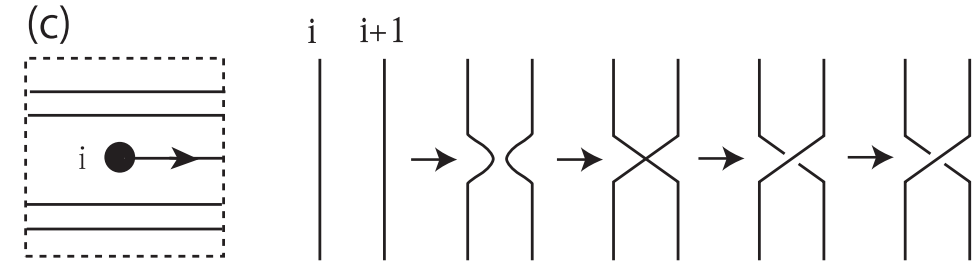

Figure 5:

not use a C-II move and a C-III move. We often use C-I-M2 moves and C-I-R2 moves in this paper.

Let $\Gamma$ be a chart, and $m$ a label of $\Gamma$. The 'subgraph' $\Gamma_{m}$ of $\Gamma$ consists of all the edges of label $m$ and their vertices. An edge of $\Gamma$ is the closure of a connected component of the set obtained by taking out all white vertices and crossings from $\Gamma$. On the other hand, we assume that

an edge of $\Gamma_{m}$ is the closure of a connected component of the set obtained by taking out all white vertices from $\Gamma_{m}$.

Thus any vertex of $\Gamma_{m}$ is a black vertex or a white vertex. Hence any crossing of $\Gamma$ is not considered as a vertex of $\Gamma_{m}$.

In this paper for a set $X$ in a space we denote the interior of $X$, the boundary of $X$ and the closure of $X$ by $\operatorname{Int} X, \partial X$ and $C l(X)$ respectively. 

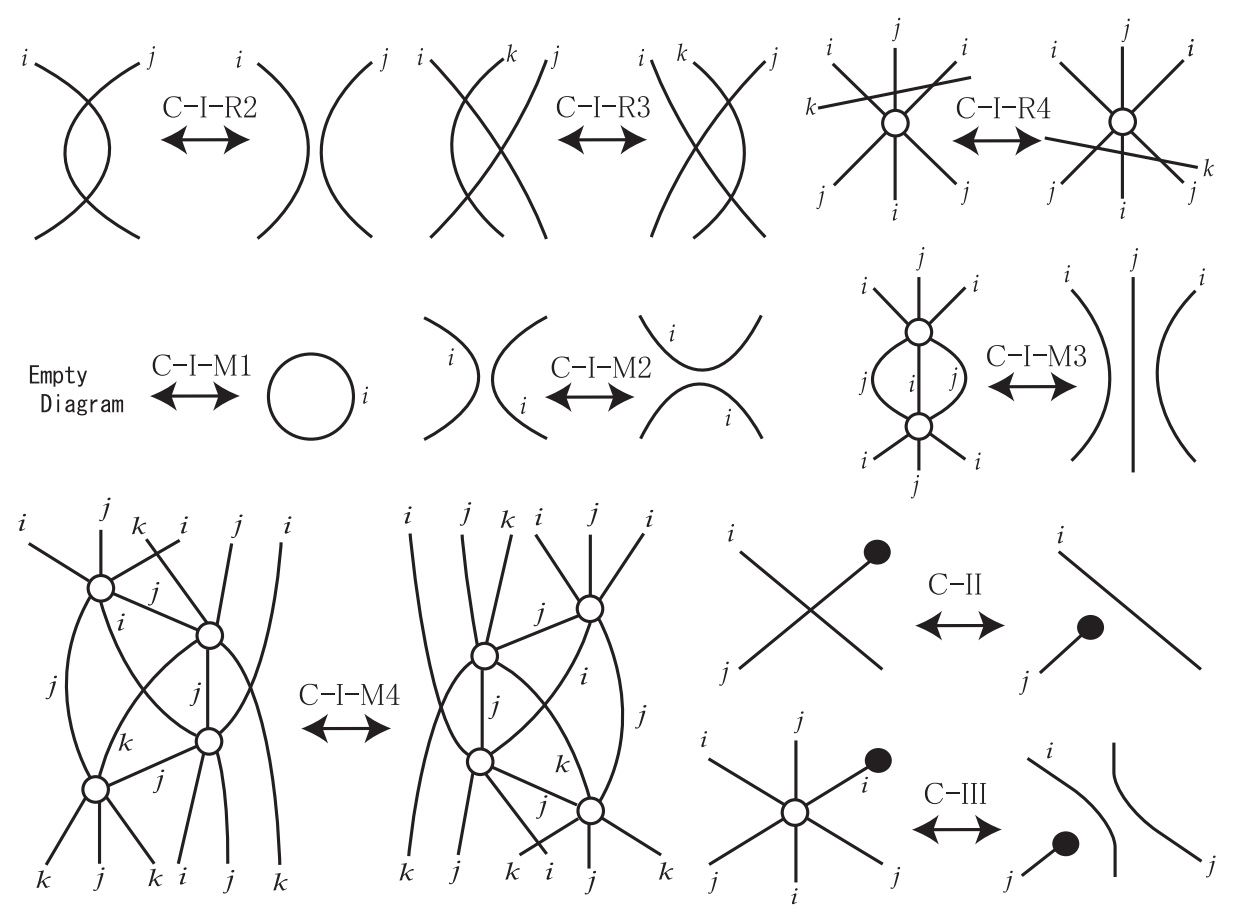

Figure 6: For the C-III move, the black vertex in the left figure is not contained in a middle edge of three consecutive edges oriented inward or outward.

\section{Separating Systems}

In this paper we assume that every chart is in the plane.

For a subset $X$ of a chart $\Gamma$, let

$$
\mathbb{B W}(X)=\text { the set of all the black and white vertices of } \Gamma \text { in } X \text {. }
$$

A disk $D$ is in general position with respect to a chart $\Gamma$ provided that

(i) $\partial D$ does not intersect the set of crossings nor $\mathbb{B} \mathbb{W}(\Gamma)$, and

(ii) if an edge of $\Gamma$ intersects $\partial D$, then the edge intersects $\partial D$ transversely.

Let $D$ be a disk. A simple arc $\ell$ is called a proper arc of $D$ provided that $\ell \cap \partial D=\partial \ell$. Let $L$ be a simple $\operatorname{arc}$ on $\partial D$. A proper $\operatorname{arc} \ell$ of $D$ is called a $(D, L)$-arc provided that $\partial \ell \subset L$.

Let $\Gamma$ be a chart, and $k$ a label of $\Gamma$. A simple arc in an edge of $\Gamma_{k}$ is called an arc of label $k$. 
Let $D$ be a disk in general position with respect to a chart $\Gamma$, and $L$ a simple arc on $\partial D$. A $(D, L)$-arc $\ell$ of label $k$ is said to be reducible if for the subarc $L^{\prime}$ of $L$ with $\partial L^{\prime}=\partial \ell$ we have $\operatorname{Int} L^{\prime} \cap\left(\Gamma_{k-1} \cup \Gamma_{k} \cup \Gamma_{k+1}\right)=\emptyset$.

Let $k$ be a positive integer. Let $D^{-}$and $D^{+}$be disks in general position with respect to a chart $\Gamma$ such that $J=D^{-} \cap D^{+}$is an arc. The triplet $\left(D^{-}, D^{+}, J\right)$ is called a separating system at label $k$ for the chart $\Gamma$ provided that

(i) $D^{-} \cap \mathbb{B} \mathbb{W}\left(\bigcup_{i=k+1}^{\infty} \Gamma_{i}\right)=\emptyset$,

(ii) $D^{+} \cap \mathbb{B} \mathbb{W}\left(\bigcup_{i=1}^{k} \Gamma_{i}\right)=\emptyset$,

(iii) $J \cap \Gamma_{k}=\emptyset$, and

(iv) $C l\left(\partial D^{+}-J\right) \cap \Gamma=\emptyset$.

Lemma 6. Let $\left(D^{-}, D^{+}, J\right)$ be a separating system at label $k$ for a chart $\Gamma$. Then the following hold:

(a) If there exists a $\left(D^{+}, J\right)$-arc of label less than $k$, then there exists a reducible $\left(D^{+}, J\right)$-arc of label less than $k$.

(b) If there exists a $\left(D^{-}, J\right)$-arc of label greater than $k$, then there exists a reducible $\left(D^{-}, J\right)$-arc of label greater than $k$.

Proof. We show Statement (a). Suppose that there exists a $\left(D^{+}, J\right)$-arc of label less than $k$. Let $\mathbb{S}$ be the set of all $\left(D^{+}, J\right)$-arcs of label less than $k$. For each $\ell \in \mathbb{S}$ let $P_{\ell}$ be the subarc of $J$ with $\partial P_{\ell}=\partial \ell$. Let

$$
m(\ell)=\text { the number of points } \operatorname{in} \operatorname{Int}\left(P_{\ell}\right) \cap\left(\bigcup_{i=1}^{k} \Gamma_{i}\right) .
$$

Let $\ell_{0}$ be an element in $\mathbb{S}$ with

$$
m\left(\ell_{0}\right)=\min \{m(\ell) \mid \ell \in \mathbb{S}\} .
$$

Let $j$ be the label of $\ell_{0}$. We have $j \leq k-1$. Let

$$
s=\text { the number of points } \operatorname{in} \operatorname{Int}\left(P_{\ell_{0}}\right) \cap\left(\Gamma_{j-1} \cup \Gamma_{j} \cup \Gamma_{j+1}\right) .
$$

We show that $s=0$ by contradiction. Suppose that $s>0$. Let $D_{0}$ be the disk in $D^{+}$bounded by $\ell_{0} \cup P_{\ell_{0}}$. Let $\ell_{1}$ be a connected component of $D_{0} \cap\left(\Gamma_{j-1} \cup \Gamma_{j} \cup \Gamma_{j+1}\right)$ different from $\ell_{0}$. Since $j+1 \leq(k-1)+1=k$ and since there do not exist any black vertices nor white vertices of $\bigcup_{i=1}^{k} \Gamma_{i}$ in $D^{+}$by Condition (ii) of a separating sysytem, the $\operatorname{arc} \ell_{1}$ is a proper $\operatorname{arc}$ of $D_{0}$. Since $\ell_{0} \cap \ell_{1}=\emptyset$, the $\operatorname{arc} \ell_{1}$ is a $\left(D_{0}, P_{\ell_{0}}\right)$ arc. Hence $\ell_{1}$ is a $\left(D^{+}, J\right)$-arc. Let $p$ be the label of $\ell_{1}$. Now $J \cap \Gamma_{k}=\emptyset$ implies $p \neq k$. Hence we have $p<k$. Thus $\ell_{1} \in \mathbb{S}$. Since $P_{\ell_{1}} \cap \ell_{0}=\emptyset$, we have

$$
m\left(\ell_{1}\right) \leq m\left(\ell_{0}\right)-2 .
$$

This contradicts that $m\left(\ell_{0}\right)$ is minimal. Hence we have $s=0$. Therefore

$$
\operatorname{Int}\left(P_{\ell_{0}}\right) \cap\left(\Gamma_{j-1} \cup \Gamma_{j} \cup \Gamma_{j+1}\right)=\emptyset .
$$


This means that $\ell_{0}$ is a desired reducible $\left(D^{+}, J\right)$-arc. This proves Statement (a).

Similarly we can show Statement (b).

Let $\Gamma$ be a chart, and $e_{1}$ and $e_{2}$ edges of label $m$ (possibly $e_{1}=e_{2}$ ). Let $\alpha$ be an arc such that

(i) $\partial \alpha$ consists of a point in $e_{1}$ and a point in $e_{2}$, and

(ii) $\operatorname{Int}(\alpha)$ transversely intersects edges of $\Gamma$ (see Figure $7(\mathrm{a})$ ).

Let $D$ be a regular neighborhood of the arc $\alpha$. Let $\gamma_{1}=e_{1} \cap D$ and $\gamma_{2}=e_{2} \cap D$. Then $\gamma_{1}$ and $\gamma_{2}$ are proper arcs of $D$ and they split the disk $D$ into three disks. Let $E$ be the one of the three disks with $E \supset \alpha$ (see Figure 7(b)). A chart $\Gamma^{\prime}$ is obtained from $\Gamma$ by a surgery along $\alpha$ provided that

(iii) $\Gamma_{m}^{\prime}=\left(\Gamma_{m}-\left(\gamma_{1} \cup \gamma_{2}\right)\right) \cup C l\left(\partial E-\left(\gamma_{1} \cup \gamma_{2}\right)\right)$, and

(iv) $\Gamma_{i}^{\prime}=\Gamma_{i}(i \neq m)($ see Figure $7(\mathrm{c}))$.
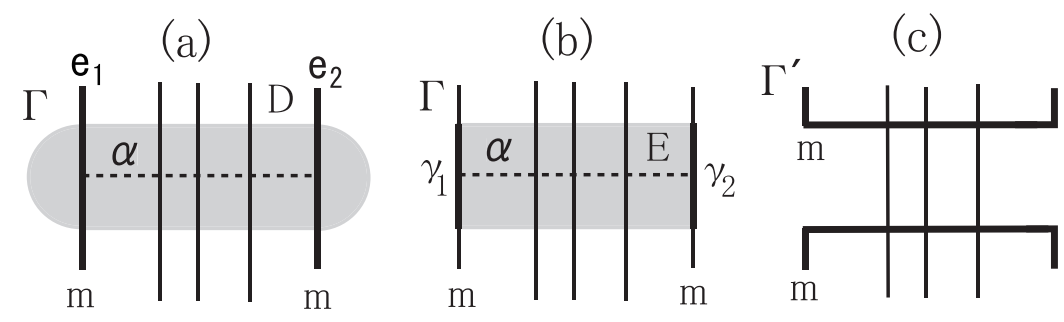

Figure 7:

Let $k$ be a positive integer. Let $\Gamma$ and $\Gamma^{*}$ be charts. We write $\Gamma^{*} \stackrel{k}{\sim} \Gamma$ provided that

(i) the chart $\Gamma^{*}$ is obtained from $\Gamma$ by applying C-I-M2 moves, C-I-R2 moves and ambient isotopies of the plane, and

(ii) $\Gamma_{k}$ is a subset of $\Gamma_{k}^{*}$.

For a positive integer $k$ and a chart $\Gamma$, let

$$
\operatorname{Fix}\left(\Gamma_{k} ; \Gamma\right)=\left\{\Gamma^{*} \mid \Gamma^{*} \stackrel{k}{\sim} \Gamma\right\} .
$$

Remark. The relation $\Gamma^{*} \stackrel{k}{\sim} \Gamma$ implies that $\Gamma^{*}$ and $\Gamma$ are same C-type.

Lemma 7. Let $\left(D^{-}, D^{+}, J\right)$ be a separating system at label $k$ for a chart $\Gamma$. Then there exists a chart $\Gamma^{\prime} \in \operatorname{Fix}\left(\Gamma_{k} ; \Gamma\right)$ such that

(a) the chart $\Gamma^{\prime}$ is obtained from $\Gamma$ by applying surgeries along subarcs of $J$, and

(b) the chart $\Gamma^{\prime}$ does not possess any $\left(D^{-}, J\right)$-arcs of label greater than $k$ nor $\left(D^{+}, J\right)$-arcs of label less than $k$. 
Proof. Let $\mathbb{S}$ be the set of all charts obtained from $\Gamma$ by applying surgeries along subarcs of $J$. Since $J \cap \Gamma_{k}=\emptyset$ by Condition (ii) of a separating system, we have that $\Gamma_{k}^{*}=\Gamma_{k}$ for each chart $\Gamma^{*} \in \mathbb{S}$. Thus for each chart $\Gamma^{*} \in \mathbb{S}$ we have that $\Gamma^{*} \in \operatorname{Fix}\left(\Gamma_{k} ; \Gamma\right)$ and that $\left(D^{-}, D^{+}, J\right)$ is a separating system at label $k$ for $\Gamma^{*}$. For each chart $\Gamma^{*} \in \mathbb{S}$, let

$$
\begin{aligned}
n\left(\Gamma^{*}\right) & =\text { the number of }\left(D^{+}, J\right) \text {-arcs of label less than } k \\
& + \text { the number of }\left(D^{-}, J\right) \text {-arcs of label greater than } k .
\end{aligned}
$$

Let $\Gamma^{\prime}$ be a chart in $\mathbb{S}$ such that

$$
n\left(\Gamma^{\prime}\right)=\min \left\{n\left(\Gamma^{*}\right) \mid \Gamma^{*} \in \mathbb{S}\right\} .
$$

We show that $n\left(\Gamma^{\prime}\right)=0$ by contradiction. Suppose that $n\left(\Gamma^{\prime}\right)>0$. Then by Lemma 6 the chart $\Gamma^{\prime}$ possesses a reducible $\left(D^{-}, J\right)$-arc of label greater than $k$ or a reducible $\left(D^{+}, J\right)$-arc of label less than $k$, say $\ell$. Let $P_{\ell}$ be the subarc of $J$ with $\partial P_{\ell}=\partial \ell$. Let $\Gamma^{\prime \prime}$ be a chart obtained from $\Gamma^{\prime}$ by applying a surgery along the subarc $P_{\ell}$. Then we have $\Gamma^{\prime \prime} \in \mathbb{S}$ and

$$
n\left(\Gamma^{\prime \prime}\right) \leq n\left(\Gamma^{\prime}\right)-1 \text {. }
$$

This contradicts that $n\left(\Gamma^{\prime}\right)$ is minimal. Therefore $n\left(\Gamma^{\prime}\right)=0$. The chart $\Gamma^{\prime}$ is a desired chart.

\section{Movable Disks}

Let $D$ be a disk in general position with respect to a chart $\Gamma$. The disk $D$ is called a movable disk at label $k$ with respect to the chart $\Gamma$ provided that

(i) $D \cap \mathbb{B} \mathbb{W}\left(\bigcup_{i=1}^{k} \Gamma_{i}\right)=\emptyset$, and

(ii) $\partial D \cap\left(\bigcup_{i=1}^{k+1} \Gamma_{i}\right)=\emptyset$.

Let $\Gamma$ be a chart, and $m$ a label of $\Gamma$. A hoop is a closed edge of $\Gamma$ without vertices (hence without crossings, neither). A ring is a closed edge of $\Gamma_{m}$ containing a crossing but not containing any white vertices.

Lemma 8. Let $\left(D^{-}, D^{+}, J\right)$ be a separating system at label $k$ for a chart $\Gamma$. If $\partial\left(D^{-} \cup D^{+}\right) \cap \Gamma_{k+1}=\emptyset$, then there exists a chart $\Gamma^{\prime} \in F i x\left(\Gamma_{k} ; \Gamma\right)$ such that

(a) the chart $\Gamma^{\prime}$ is obtained from $\Gamma$ by applying surgeries along subarcs of $J$, and

(b) $D^{+}$is a movable disk at label $k$ with respect to $\Gamma^{\prime}$.

Proof. By Lemma 7 there exists a chart $\Gamma^{\prime} \in F i x\left(\Gamma_{k} ; \Gamma\right)$ obtained from $\Gamma$ by applying surgeries along subarcs of $J$ such that

(1) $\Gamma^{\prime}$ does not possess any $\left(D^{-}, J\right)$-arcs of label greater than $k$ nor $\left(D^{+}, J\right)$-arcs of label less than $k$.

Thus by Condition (ii), (iii) and (iv) of a separating system, 
(2) $D^{+} \cap \mathbb{B} \mathbb{W}\left(\bigcup_{i=1}^{k} \Gamma_{i}^{\prime}\right)=D^{+} \cap \mathbb{B} \mathbb{W}\left(\bigcup_{i=1}^{k} \Gamma_{i}\right)=\emptyset$,

(3) $J \cap \Gamma_{k}^{\prime}=J \cap \Gamma_{k}=\emptyset$,

(4) $C l\left(\partial D^{+}-J\right) \cap \Gamma^{\prime}=C l\left(\partial D^{+}-J\right) \cap \Gamma=\emptyset$.

Hence any connected component of $D^{+} \cap\left(\bigcup_{i=1}^{k} \Gamma_{i}^{\prime}\right)$ is a hoop, a ring or a $\left(D^{+}, J\right)$-arc of label less than $k$. Since $\Gamma^{\prime}$ does not possess any $\left(D^{+}, J\right)$-arcs of label less than $k$ by (1), we have

(5) $J \cap\left(\bigcup_{i=1}^{k} \Gamma_{i}^{\prime}\right)=\emptyset$.

Since the chart $\Gamma^{\prime}$ is obtained from $\Gamma$ by applying surgeries along subarcs of $J$, we have

(6) $\partial\left(D^{-} \cup D^{+}\right) \cap \Gamma_{k+1}^{\prime}=\partial\left(D^{-} \cup D^{+}\right) \cap \Gamma_{k+1}=\emptyset$, and

(7) $D^{-} \cap \mathbb{B} \mathbb{W}\left(\bigcup_{i=k+1}^{\infty} \Gamma_{i}^{\prime}\right)=D^{-} \cap \mathbb{B} \mathbb{W}\left(\bigcup_{i=k+1}^{\infty} \Gamma_{i}\right)=\emptyset$ by Condition (i) of a separating system.

Hence any connected component of $D^{-} \cap \Gamma_{k+1}^{\prime}$ is a hoop, a ring or a $\left(D^{-}, J\right)$-arc. Since $\Gamma^{\prime}$ does not possess any $\left(D^{-}, J\right)$-arcs of label greater than $k$ by $(1)$, we have $J \cap \Gamma_{k+1}^{\prime}=\emptyset$. Thus (4) and (5) imply

$$
\partial D^{+} \cap\left(\bigcup_{i=1}^{k+1} \Gamma_{i}^{\prime}\right)=\emptyset .
$$

Therefore $D^{+}$is a movable disk at label $k$ with respect to $\Gamma^{\prime}$.

Let $E$ be a disk in general position with respect to a chart $\Gamma$. The disk $E$ is called a $c$-disk at label $k$ with respect to $\Gamma$ provided that in $\operatorname{Int} E$ there exist mutually disjoint movable disks $D_{1}, D_{2}, \cdots, D_{s}$ at label $k$ with respect to the chart $\Gamma$ and a connected component $W$ of $E-\left(\Gamma_{k}-\bigcup_{i=1}^{s} D_{i}\right)$ (see Figure 8) such that

(i) $W \supset \partial E$,

(ii) $W \supset \bigcup_{i=1}^{s} D_{i}$, and

(iii) $W \supset E \cap \mathbb{B} \mathbb{W}\left(\bigcup_{i=k+1}^{\infty} \Gamma_{i}\right)$.

We call $W$ the principal domain of the c-disk $E$. We also call the movable disks $D_{1}, D_{2}, \cdots, D_{s}$ associated movable disks of the c-disk $E$.

Lemma 9. Let $E$ be a c-disk at label $k$ with respect to a chart $\Gamma$. Let $p \in \partial E-\Gamma$. Then there exists a separating system $\left(D^{-}, D^{+}, J\right)$ at label $k$ for the chart $\Gamma$ with $E=D^{-} \cup D^{+}$and $p \in \partial D^{+}-J$.

Proof. Let $W$ be the principal domain of the c-disk $E$ and $D_{1}, D_{2}, \cdots, D_{s}$ associated movable disks of $E$. Suppose that

$$
\left(W-\bigcup_{i=1}^{s} D_{i}\right) \cap \mathbb{B} \mathbb{W}\left(\bigcup_{i=k+1}^{\infty} \Gamma_{i}\right)=\left\{w_{s+1}, w_{s+2}, \ldots, w_{t}\right\} .
$$

For each $i=s+1, s+2, \ldots, t$, let $D_{i}$ be a regular neighbourhood of $w_{i}$ in $W$. Then by Condition (iii) of a c-disk we have 

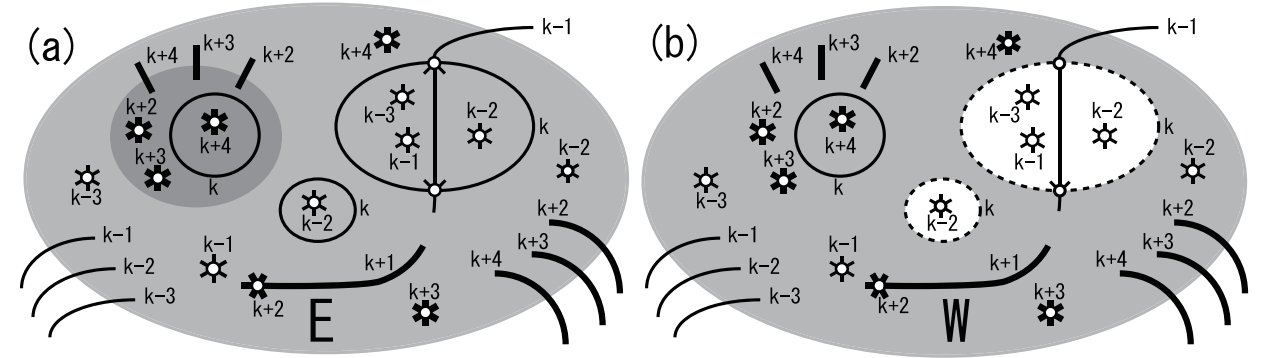

Figure 8: (a) The dark disk is a movable disk in a c-disk. (b) The gray 'disk with two holes' is the principal domain of the c-disk.

(1) $E \cap \mathbb{B} \mathbb{W}\left(\bigcup_{i=k+1}^{\infty} \Gamma_{i}\right) \subset \bigcup_{i=1}^{t} D_{i}$.

Since $W$ is a connected component of $E-\left(\Gamma_{k}-\bigcup_{i=1}^{s} D_{i}\right)$ by the condition of a c-disk, we have

$$
\left(W-\bigcup_{i=1}^{s} D_{i}\right) \cap \Gamma_{k}=\emptyset .
$$

Hence there exist mutually disjoint simple $\operatorname{arcs} \ell_{1}, \ell_{2}, \cdots, \ell_{t}$ in $W$ (see Figure $9($ a)) such that

(2) for each $i=1,2, \cdots, t, \ell_{i} \cap \Gamma_{k}=\emptyset$, and $\partial \ell_{i} \cap \Gamma=\emptyset$,

(3) for each $i=1,2, \cdots, t$, the $\operatorname{arc} \ell_{i}$ does not contain any white vertices, black vertices nor crossings of $\Gamma$, and the arc $\ell_{i}$ intersects edges of $\Gamma$ transversely,

(4) $\left(\bigcup_{i=1}^{t} \operatorname{Int}\left(\ell_{i}\right)\right) \cap\left(\bigcup_{i=1}^{t} D_{i}\right)=\emptyset$,

(5) for each $i=1,2, \cdots, t-1$, the $\operatorname{arc} \ell_{i}$ connects a point on $\partial D_{i}$ and a point on $\partial D_{i+1}$, and

(6) the arc $\ell_{t}$ connects a point on $\partial D_{t}$ and the point $p$.

Let $D^{+}$be a regular neighbourhood of $\bigcup_{i=1}^{t}\left(D_{i} \cup \ell_{i}\right)$ in $E$ and $D^{-}=C l\left(E-D^{+}\right)$. Then $D^{-}$and $D^{+}$are disks with $E=D^{-} \cup D^{+}$. Since $E \cap \mathbb{B} \mathbb{W}\left(\bigcup_{i=k+1}^{\infty} \Gamma_{i}\right) \subset$ $\bigcup_{i=1}^{t} D_{i} \subset D^{+}$by (1), we have

$$
\left.D^{-} \cap \mathbb{B} \mathbb{W}\left(\bigcup_{i=k+1}^{\infty} \Gamma_{i}\right)=\emptyset \text { (see Figure } 9(\mathrm{~b})\right) .
$$

Since $w_{s+1}, w_{s+2}, \ldots, w_{t} \in W-\bigcup_{i=1}^{s} D_{i} \subset E-\Gamma_{k}$, we have $w_{s+1}, w_{s+2}, \ldots, w_{t} \notin \Gamma_{k}$. Thus

(7) none of $D_{s+1}, D_{s+2}, \cdots, D_{t}$ intersect $\bigcup_{i=1}^{k} \Gamma_{i}$.

Since none of the movable disks at label $k$ nor $D_{s+1}, D_{s+2}, \cdots, D_{t}$ intersect $\mathbb{B} \mathbb{W}\left(\bigcup_{i=1}^{k} \Gamma_{i}\right)$, we have

$$
D^{+} \cap \mathbb{B} \mathbb{W}\left(\bigcup_{i=1}^{k} \Gamma_{i}\right)=\emptyset \text {. }
$$


Let $L=D^{+} \cap \partial E$. Then $L$ is a regular neighbourhood of $p$ in $\partial E$. Since $p \in$ $\partial E-\Gamma$, we have $L \cap \Gamma=\emptyset$. Let $J=D^{-} \cap D^{+}$. Then $L=C l\left(\partial D^{+}-J\right)$. Thus $C l\left(\partial D^{+}-J\right) \cap \Gamma=L \cap \Gamma=\emptyset$. Since for each $i=1,2, \cdots, t, \partial D_{i} \cap \Gamma_{k}=\emptyset$ by (2), (7) and Condition (ii) of a movable disk, we have $J \cap \Gamma_{k}=\emptyset$. Therefore the triplet $\left(D^{-}, D^{+}, J\right)$ is a desired separating system at label $k$ for $\Gamma$.

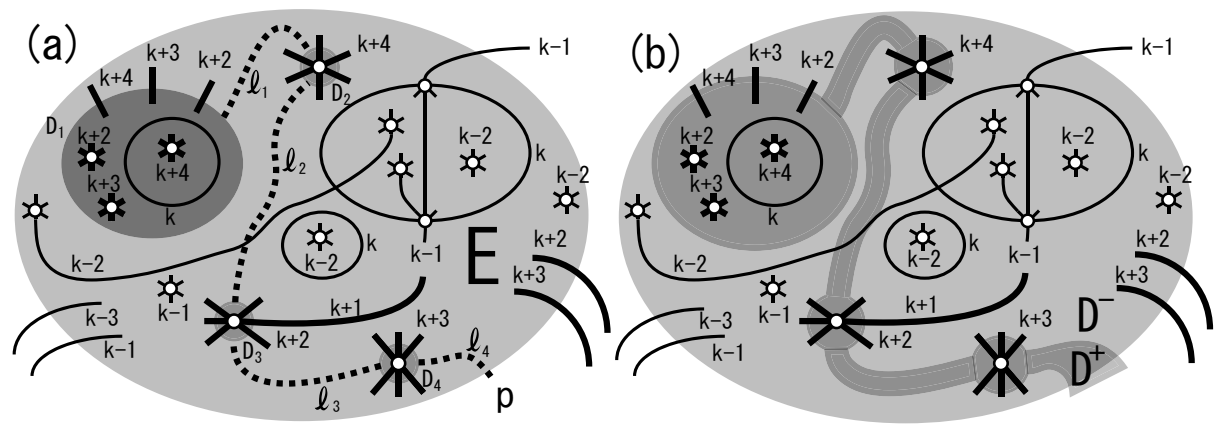

Figure 9: (a) The dark disk is a movable disk.

\section{5. $\omega_{k}$-minimal Charts}

Let $\Gamma$ be a chart. Let $\Gamma^{*}$ be a chart in $\operatorname{Fix}\left(\Gamma_{k} ; \Gamma\right)$. We denote $\Gamma^{*} \stackrel{k}{\approx} \Gamma$ provided that

for any complementary domain $U$ of $\Gamma_{k}$, the domain $U$ contains mutually disjoint movable disks $D_{1}, D_{2}, \cdots, D_{s}$ at label $k$ with respect to $\Gamma^{*}$ with $U \cap\left(\Gamma_{k}^{*}-\Gamma_{k}\right) \subset \bigcup_{i=1}^{s} D_{i}$.

The movable disks $D_{1}, D_{2}, \cdots, D_{s}$ are called basic movable disks at label $k$ with respect to $U$ and $\Gamma^{*}$. Let

$$
\Omega\left(\Gamma_{k} ; \Gamma\right)=\left\{\Gamma^{*} \mid \Gamma^{*} \stackrel{k}{\approx} \Gamma\right\}
$$

Since $\Gamma \in \Omega\left(\Gamma_{k} ; \Gamma\right)$, we have $\Omega\left(\Gamma_{k} ; \Gamma\right) \neq \emptyset$.

Let $\Gamma$ be a chart, $k$ a label of $\Gamma$, and $\Gamma^{\prime} \in \Omega\left(\Gamma_{k} ; \Gamma\right)$. Let $U$ be a complementary domain of $\Gamma_{k}$, and $D$ a movable disk at label $k$ in $U$ with respect to $\Gamma^{\prime}$. Let $F=C l(U)$. Let $p \in \partial F-\bigcup_{i \neq k} \Gamma_{i}^{\prime}$ and $q \in \partial D$. Suppose that there exists a simple arc $\alpha$ in $F$ connecting the two points $p$ and $q$ with $\alpha \cap \Gamma^{\prime}=p$ and $\alpha \cap$ $D=q$ (see Figure 10(a)). Then we can shift the movable disk $D$ at label $k$ to another complementary domain of $\Gamma_{k}$ as follows (see Figure 10): Let $N_{1}$ be a regular neighbourhood of $D \cup \alpha$ in $F$. Let $N_{2}$ be a regular neighbourhood of $N_{1}$ in $F$ and $N_{3}$ a regular neighbourhood of $N_{2}$ in $F$. For each $i=1,2,3$ let $\beta_{i}=N_{i} \cap \partial F$ and $\gamma_{i}=C l\left(\partial N_{i}-\beta_{i}\right)$ (see Figure 10(b)). Let $\Gamma^{\prime \prime}$ be a chart with

$$
\Gamma_{j}^{\prime \prime}= \begin{cases}\left(\Gamma_{k}^{\prime}-\beta_{3}\right) \cup\left(\gamma_{3} \cup \partial N_{1}\right) & \text { if } j=k, \\ \Gamma_{j}^{\prime} & \text { otherwise. }\end{cases}
$$


Then $\Gamma^{\prime}$ is C-move equivalent to $\Gamma^{\prime \prime}$ by modifying $\partial F$ by C-I-R 2 moves along $\gamma_{2}$ and a C-I-M2 move (see Figure 10(c)). Let $N$ be a regular neighbourhood of $N_{1}$. Then $N$ is a movable disk at label $k$ with respect to $\Gamma^{\prime \prime}$. In a regular neighbourhood of $N_{3}$ we can modify the arc $\gamma_{3}$ to the arc $\beta_{3}$ by an ambient isotopy keeping $\partial \gamma_{3}$ fixed. Let $\Gamma^{*}$ be the resulting chart and $D^{*}$ the disk modified from the movable disk $N$ (see Figure 10(d)). Then $\Gamma^{*}$ is in $F i x\left(\Gamma_{k} ; \Gamma\right)$ and $D^{*}$ is a movable disk at label $k$ with respect to $\Gamma^{*}$. Now $\Gamma_{k}^{*}$ is the union of $\Gamma_{k}^{\prime}$ and a ring in the movable disk $D^{*}$. Thus $\Gamma^{*} \stackrel{k}{\approx} \Gamma$. We say that $\Gamma^{*}$ is obtained from $\Gamma^{\prime}$ by shifting the movable disk $D$ to the outside of $C l(U)$ along the arc $\alpha$ and that $D^{*}$ is a movable disk induced from the movable disk $D$ circled by a ring of label $k$.
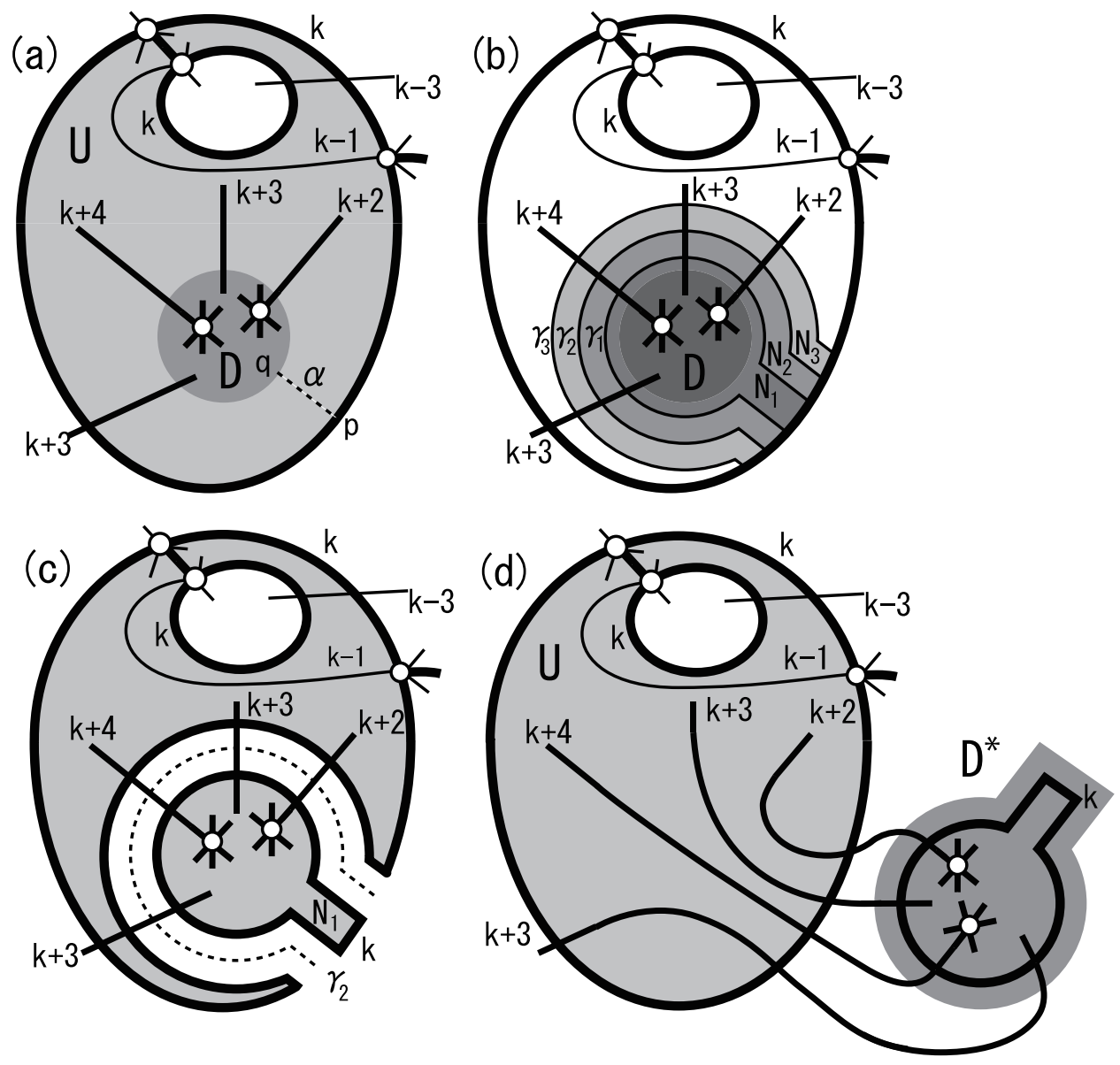

Figure 10: Shifting a movable disk.

Let $\Gamma$ be a chart and $k$ a label of $\Gamma$. Let $T$ be a maximal tree of the dual graph 
of $\Gamma_{k}$. Namely

(i) each vertex $v$ of the tree $T$ corresponds to a complementary domain $U_{v}$ of $\Gamma_{k}$, and

(ii) each edge $e$ of the tree $T$ with $\partial e=\left\{v_{1}, v_{2}\right\}$ corresponds to an edge $e_{\Gamma}$ of $\Gamma_{k}$ with $e_{\Gamma} \subset C l\left(U_{v_{1}}\right) \cap C l\left(U_{v_{2}}\right)$.

The tree $T$ is called a dual tree with respect to $\Gamma_{k}$.

Let $\Gamma$ be a chart and $k$ a label of $\Gamma$. Let $T$ be a dual tree with respect to $\Gamma_{k}$. Let $v_{0}$ be the vertex of $T$ which corresponds to the unbounded complementary domain of $\Gamma_{k}$. Let $V(T)$ be the set of all vertices of $T$. For each vertex $v \in V(T)$, let $T\left(v, v_{0}\right)$ be the path in $T$ connecting $v$ and $v_{0}$. Let

$$
\text { length }(v ; T)=\text { the number of edges in } T\left(v, v_{0}\right) \text {. }
$$

We have length $\left(v_{0} ; T\right)=0$. For each chart $\Gamma^{*} \in \Omega\left(\Gamma_{k} ; \Gamma\right)$ and $v \in V(T)$, let

$$
\begin{aligned}
& \text { weight }_{k}\left(v ; \Gamma^{*}\right)= \begin{cases}0 & \text { if } U_{v} \cap \mathbb{B} \mathbb{W}\left(\bigcup_{i=k+1}^{\infty} \Gamma_{i}^{*}\right)=\emptyset, \\
1 & \text { otherwise, }\end{cases} \\
& \omega_{k}\left(\Gamma^{*}, T\right)=\sum_{v \in V(T)} \text { weight }_{k}\left(v ; \Gamma^{*}\right) \times \text { length }(v ; T) .
\end{aligned}
$$

A chart $\Gamma^{\prime} \in \Omega\left(\Gamma_{k} ; \Gamma\right)$ is $\omega_{k}$-minimal if there exists a dual tree $T$ with respect to $\Gamma_{k}$ such that $\omega_{k}\left(\Gamma^{\prime}, T\right)=\min \left\{\omega_{k}\left(\Gamma^{*}, T\right) \mid \Gamma^{*} \in \Omega\left(\Gamma_{k} ; \Gamma\right)\right\}$.

Lemma 10. Let $\Gamma$ be a chart which is zero at label $k$. If a chart $\Gamma^{\prime} \in \Omega\left(\Gamma_{k} ; \Gamma\right)$ is $\omega_{k}$-minimal, then there exists a dual tree $T$ with respect to $\Gamma_{k}$ with $\omega_{k}\left(\Gamma^{\prime}, T\right)=0$.

Proof. Suppose that for a dual tree $T$ with respect to $\Gamma_{k}$, we have

$$
\omega_{k}\left(\Gamma^{\prime}, T\right)=\min \left\{\omega_{k}\left(\Gamma^{*}, T\right) \mid \Gamma^{*} \in \Omega\left(\Gamma_{k} ; \Gamma\right)\right\} .
$$

We shall show $\omega_{k}\left(\Gamma^{\prime}, T\right)=0$ by contradiction. Suppose that $\omega_{k}\left(\Gamma^{\prime}, T\right)>0$. Let $V(T)$ be the set of all vertices of $T$ and

$$
\mathbb{P}=\left\{v \in V(T) \mid \text { weight }_{k}\left(v ; \Gamma^{\prime}\right) \times \operatorname{length}(v ; T)>0\right\} .
$$

Then $\omega_{k}\left(\Gamma^{\prime}, T\right)>0$ implies $\mathbb{P} \neq \emptyset$. Let $v_{1}$ be a vertex in $\mathbb{P}$ such that

(1) length $\left(v_{1} ; T\right)=\max \{\operatorname{length}(v ; T) \mid v \in \mathbb{P}\}$.

Then $v_{1} \in \mathbb{P}$ implies

(2) $0<$ weight $_{k}\left(v_{1} ; \Gamma^{\prime}\right) \times \operatorname{length}\left(v_{1} ; T\right)=\operatorname{length}\left(v_{1} ; T\right)$.

Let $V_{1}$ be a complementary domain of $\Gamma_{k}$ corresponding to $v_{1}$. Since $\Gamma^{\prime} \in$ $\Omega\left(\Gamma_{k} ; \Gamma\right)$, there exist basic movable disks $D_{1}, D_{2}, \cdots, D_{s}$ at label $k$ with respect to $V_{1}$ and $\Gamma^{\prime}$ (see Figure 11(a)) such that

(3) $V_{1} \cap\left(\Gamma_{k}^{\prime}-\Gamma_{k}\right) \subset \bigcup_{i=1}^{s} D_{i}$. 
Let $\delta$ be a connected component of $\partial V_{1}$ such that a bounded complementary domain of $\delta$ contains $V_{1}$ (see Figure 11(b)). Let $N(\delta)$ be a regular neighbourhood of $\delta$ and $W=C l\left(V_{1}-N(\delta)\right.$ ) (see Figure 11(c)). Let $\ell$ be a connected component of $\partial W$ such that $W$ is contained in the disk $E$ bounded by $\ell$ (see Figure 11(c) and (d)). Then we have

$$
W \supset \partial E .
$$

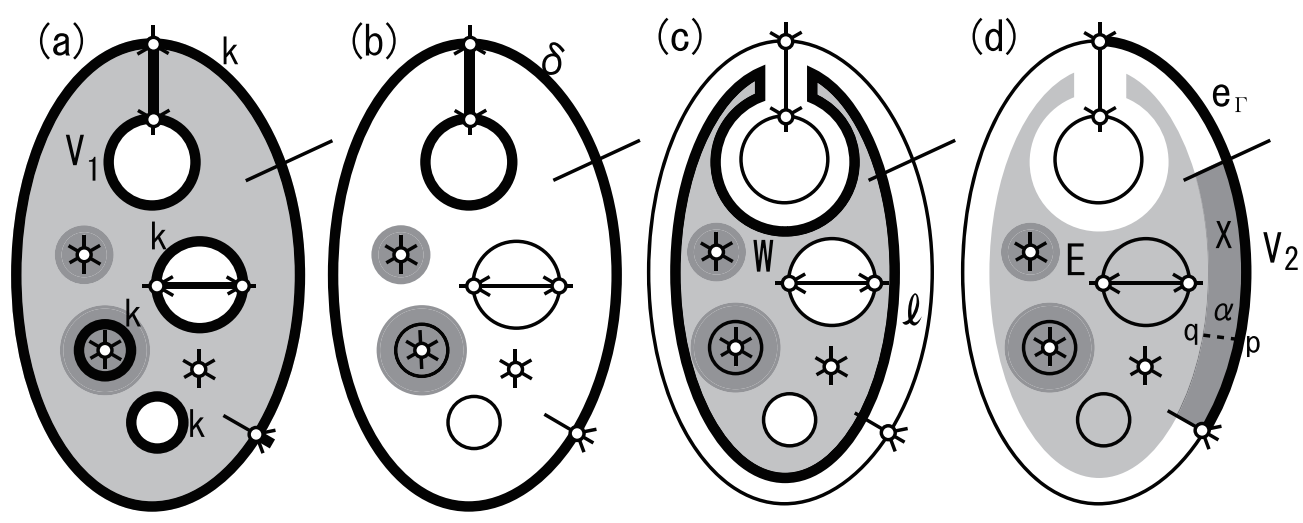

Figure 11: Dark disks are movable disks at label $k$. (a) The thicken curves are of label $k$. (b) The thicken curve is the set $\delta$. (c) The thicken curve is the simple closed curve $\ell$. (d) The thicken curve is the edge $e_{\Gamma}$. The set $X$ is a disk with $\operatorname{Int} X \cap \operatorname{Int} E=\emptyset$.

Claim. The disk $E$ is a c-disk.

Proof of Claim. Since $\Gamma^{\prime} \stackrel{k}{\approx} \Gamma$, the chart $\Gamma^{\prime}$ is zero at label $k$. Hence $\delta \subset \Gamma_{k} \subset \Gamma_{k}^{\prime}$ implies that $\delta \cap \Gamma_{k+1}^{\prime}=\emptyset$. Thus

(4) $\partial E \cap\left(\Gamma_{k}^{\prime} \cup \Gamma_{k+1}^{\prime}\right)=\emptyset$.

Now $\delta \cap\left(\bigcup_{i=1}^{s} D_{i}\right)=\emptyset$ implies $N(\delta) \cap\left(\bigcup_{i=1}^{s} D_{i}\right)=\emptyset$. Thus $V_{1} \supset \bigcup_{i=1}^{s} D_{i}$ implies

$$
W=C l\left(V_{1}-N(\delta)\right) \supset \bigcup_{i=1}^{s} D_{i} .
$$

Let $\mathbb{S}$ be the set of all connected components of $E-\left(\Gamma_{k}-\bigcup_{i=1}^{s} D_{i}\right)$ different from $W$. Then we have

(5) $E=W \cup(\bigcup\{C l(U) \mid U \in \mathbb{S}\})$.

For each domain $U \in \mathbb{S}$, let $v_{U}$ be the vertex of $T$ corresponding to $U$. Since $U$ is surrounded by $V_{1}$, we have $v_{1} \in T\left(v_{U}, v_{0}\right)$ where $v_{0}$ is the vertex of $T$ which corresponds to the unbounded complementary domain of $\Gamma_{k}$. Hence

$$
\operatorname{length}\left(v_{U} ; T\right) \geq \operatorname{length}\left(v_{1} ; T\right)+1 \text { and }
$$




$$
\text { weight }_{k}\left(v_{U} ; \Gamma^{\prime}\right)=0
$$

by the property (1) of the vertex $v_{1}$. This means

$$
U \cap \mathbb{B} \mathbb{W}\left(\bigcup_{i=k+1}^{\infty} \Gamma_{i}^{\prime}\right)=\emptyset .
$$

Since $\Gamma^{\prime} \stackrel{k}{\approx} \Gamma$, the chart $\Gamma^{\prime}$ is zero at label $k$. Thus $\Gamma_{k}^{\prime} \cap \Gamma_{k+1}^{\prime}=\emptyset$. Hence $\partial U \subset$ $\Gamma_{k} \subset \Gamma_{k}^{\prime}$ implies

$$
C l(U) \cap \mathbb{B} \mathbb{W}\left(\bigcup_{i=k+1}^{\infty} \Gamma_{i}^{\prime}\right)=\emptyset .
$$

Thus (5) implies that

$$
E \cap \mathbb{B} \mathbb{W}\left(\bigcup_{i=k+1}^{\infty} \Gamma_{i}^{\prime}\right) \subset W .
$$

Therefore the disk $E$ is a c-disk at label $k$ with respect to $\Gamma^{\prime}$. Hence Claim holds.

Let $T\left(v_{1}, v_{0}\right)$ be the path in the tree $T$ connecting $v_{1}$ and $v_{0}$. Let $e$ be the edge of $T\left(v_{1}, v_{0}\right)$ with $e \ni v_{1}$. Let $v_{2}$ be the vertex of $e$ different from $v_{1}$. Now we have

(6) length $\left(v_{2} ; T\right)=$ length $\left(v_{1} ; T\right)-1$.

Let $e_{\Gamma}$ be the edge of $\Gamma_{k}$ corresponding to the edge $e$ which connects the two vertices $v_{1}$ and $v_{2}$. Let $V_{2}$ be the complementary domain of $\Gamma_{k}$ corresponding to $v_{2}$. Then we have $e_{\Gamma} \subset C l\left(V_{1}\right) \cap C l\left(V_{2}\right)$. Let $p$ be a point in $\operatorname{Int}\left(e_{\Gamma}\right)-\bigcup_{i \neq k} \Gamma_{i}^{\prime}$ and $X$ the closure of a connected component of $\left(V_{1}-E\right)-\Gamma^{\prime}$ with $X \ni p$. Let $q$ be a point in $\partial E \cap X$ and $\alpha$ a proper simple arc in $X$ with $\alpha \cap \Gamma^{\prime}=p$ and $\alpha \cap E=q$ (see Figure 11(d)).

By Lemma 9 there exists a separating system $\left(D^{-}, D^{+}, J\right)$ at label $k$ for $\Gamma^{\prime}$ with $E=D^{-} \cup D^{+}$and $q \in D^{+} \cap \partial E$.

Now (4) implies

$$
\partial\left(D^{-} \cup D^{+}\right) \cap \Gamma_{k+1}^{\prime}=\partial E \cap \Gamma_{k+1}^{\prime}=\emptyset .
$$

Thus by Lemma 8 there exists a chart $\Gamma^{\prime \prime} \in \operatorname{Fix}\left(\Gamma_{k} ; \Gamma\right)$ obtained from $\Gamma^{\prime}$ by applying surgeries along subarcs of $J$ such that $D^{+}$is a movable disk at label $k$ with respect to $\Gamma^{\prime \prime}$.

Let $\Gamma^{*}$ be a chart obtained from $\Gamma^{\prime \prime}$ by shifting the movable disk $D^{+}$to the outside of $C l\left(V_{1}\right)$ along the arc $\alpha$ and let $D^{*}$ be a movable disk induced from the movable disk $D^{+}$circled by a ring of label $k$. Then $D^{*}$ is a new movable disk in $V_{2}$ disjoint from the old movable disks in $V_{2}$. Hence we have $\Gamma^{*} \in \Omega\left(\Gamma_{k} ; \Gamma\right)$. Now weight $_{k}\left(v_{1} ; \Gamma^{\prime}\right) \times \operatorname{length}\left(v_{1} ; T\right)=\operatorname{length}\left(v_{1} ; T\right)>0$ by $(2)$,

weight $_{k}\left(v_{1} ; \Gamma^{*}\right) \times \operatorname{length}\left(v_{1} ; T\right)=0$, weight $_{k}\left(v_{2} ; \Gamma^{*}\right) \times \operatorname{length}\left(v_{2} ; T\right)=\operatorname{length}\left(v_{2} ; T\right)=\operatorname{length}\left(v_{1} ; T\right)-1$ by $(6)$, and weight $_{k}\left(v ; \Gamma^{*}\right) \times \operatorname{length}(v ; T)=$ weight $_{k}\left(v ; \Gamma^{\prime}\right) \times \operatorname{length}(v ; T) \quad\left(v \neq v_{1}, v_{2}\right)$ imply that

$$
\omega_{k}\left(\Gamma^{*}, T\right) \leq \omega_{k}\left(\Gamma^{\prime}, T\right)-1 .
$$


This contradicts that $\Gamma^{\prime}$ is $\omega_{k}$-minimal. Therefore $\omega_{k}\left(\Gamma^{\prime}, T\right)=0$.

\section{Proof of Main Theorem}

Proof of Theorem 1. Suppose that a chart $\Gamma$ is zero at label $k$. Then there exists a chart $\Gamma^{\prime} \in \Omega\left(\Gamma_{k} ; \Gamma\right)$ such that $\Gamma^{\prime}$ is $\omega_{k}$-minimal. Since $\Gamma$ is zero at label $k$, the chart $\Gamma^{\prime}$ is zero at label $k$, too. Let $E$ be a disk containing the chart $\Gamma^{\prime}$ in its inside, i.e. Int $E \supset \Gamma^{\prime}$. By Lemma 10 there exists a dual tree $T$ with respect to $\Gamma_{k}$ with $\omega_{k}\left(\Gamma^{\prime}, T\right)=0$. Namely $\mathbb{B} \mathbb{W}\left(\bigcup_{i=k+1}^{\infty} \Gamma_{i}^{\prime}\right)$ is contained in the unbounded complementary domain $U$ of $\Gamma_{k}$. Now $U \cap \Gamma_{k}=\emptyset$. Since $\Gamma^{\prime} \in \Omega\left(\Gamma_{k} ; \Gamma\right)$, the set $U \cap\left(\Gamma_{k}^{\prime}-\Gamma_{k}\right)$ is contained in the union of basic movable disks of label $k$ with respect to $U$ and $\Gamma^{\prime}$. Furthermore, since $E \cap U$ is connected, by the same way as the one in Lemma 9 there exists a disk $D^{+}$in $E$ such that

(1) $D^{+}$is in general position with respect to $\Gamma^{\prime}$,

(2) $D^{+} \cap \partial E$ is an arc,

(3) $\partial D^{+} \cap \Gamma_{k}^{\prime}=\emptyset$, and

(4) $\mathbb{B} \mathbb{W}\left(\Gamma^{\prime}\right) \cap D^{+}=\mathbb{B} \mathbb{W}\left(\bigcup_{i=k+1}^{\infty} \Gamma_{i}^{\prime}\right)$.

Let $D^{-}=C l\left(E-D^{+}\right)$and $J=D^{-} \cap D^{+}$. Then we have

(5) $D^{-} \cap \mathbb{B} \mathbb{W}\left(\bigcup_{i=k+1}^{\infty} \Gamma_{i}^{\prime}\right)=\emptyset$ and $D^{+} \cap \mathbb{B} \mathbb{W}\left(\bigcup_{i=1}^{k} \Gamma_{i}^{\prime}\right)=\emptyset$ by (4),

(6) $C l\left(\partial D^{+}-J\right) \cap \Gamma^{\prime} \subset \partial E \cap \Gamma^{\prime}=\emptyset$ by $\operatorname{Int} E \supset \Gamma^{\prime}$,

(7) $J \cap \Gamma_{k}^{\prime} \subset \partial D^{+} \cap \Gamma_{k}^{\prime}=\emptyset$ by (3).

Hence the triplet $\left(D^{-}, D^{+}, J\right)$ is a separating system at label $k$ for $\Gamma^{\prime}$. By Lemma 7 there exists a chart $\Gamma^{\prime \prime} \in \operatorname{Fix}\left(\Gamma_{k} ; \Gamma\right)$ obtained from $\Gamma^{\prime}$ by applying surgeries along subarcs of $J$ such that

(8) $\Gamma_{k}^{\prime \prime}=\Gamma_{k}^{\prime}$,

(9) $\Gamma^{\prime \prime}$ does not possess any $\left(D^{+}, J\right)$-arcs of label less than $k$ nor $\left(D^{-}, J\right)$-arcs of label greater than $k$,

(10) $D^{-} \cap \mathbb{B} \mathbb{W}\left(\bigcup_{i=k+1}^{\infty} \Gamma_{i}^{\prime \prime}\right)=\emptyset$ and $D^{+} \cap \mathbb{B} \mathbb{W}\left(\bigcup_{i=1}^{k} \Gamma_{i}^{\prime \prime}\right)=\emptyset$ by $(5)$.

Now $\Gamma^{\prime \prime} \in \operatorname{Fix}\left(\Gamma_{k} ; \Gamma\right)$ implies that

(11) $\Gamma^{\prime \prime}$ is zero at label $k$.

Hence $\Gamma_{k}^{\prime \prime} \cap \Gamma_{k+1}^{\prime \prime}=\emptyset$. Thus (10) and $\partial\left(D^{-} \cup D^{+}\right) \cap \Gamma^{\prime \prime}=\partial E \cap \Gamma^{\prime \prime}=\emptyset$ imply that

(12) for each label $i$ with $i \leq k$ if a connected component of $\Gamma_{i}^{\prime \prime} \cap D^{+}$intersects $J$, then the component is a $\left(D^{+}, J\right)$-arc,

(13) for each label $i$ with $k<i$ if a connected component of $\Gamma_{i}^{\prime \prime} \cap D^{-}$intersects $J$, then the component is a $\left(D^{-}, J\right)$-arc. 
Since $J \cap \Gamma_{k}^{\prime \prime}=J \cap \Gamma_{k}^{\prime}=\emptyset$ by (7) and (8), we have $J \cap \Gamma^{\prime \prime}=\emptyset$ by (9), (12) and (13). Thus $\left(\partial D^{+}-J\right) \subset \partial E$ implies

$$
\partial D^{+} \cap \Gamma^{\prime \prime}=\emptyset \text {. }
$$

Let $\Gamma^{*}=D^{-} \cap \Gamma^{\prime \prime}$ and $\Gamma^{* *}=D^{+} \cap \Gamma^{\prime \prime}$. Then $\Gamma^{\prime \prime}$ is the product of $\Gamma^{*}$ and $\Gamma^{* *}$. Since $\Gamma^{\prime \prime}$ is zero at label $k$ by (11), there exist two labels $i$ and $j$ with $i \leq k<j$, $w\left(\Gamma_{i}^{\prime \prime}\right) \neq 0$ and $w\left(\Gamma_{j}^{\prime \prime}\right) \neq 0$. Namely $w\left(\Gamma^{*}\right)>0$ and $w\left(\Gamma^{* *}\right)>0$. Now (10) implies

$$
\begin{aligned}
& w\left(\Gamma_{i}^{*}\right)=0 \text { for all label } i \text { with } k<i, \text { and } \\
& w\left(\Gamma_{i}^{* *}\right)=0 \text { for all label } i \text { with } i \leq k .
\end{aligned}
$$

Therefore $\Gamma^{\prime \prime}$ is separable at label $k$. It is clear that $\Gamma^{\prime \prime}$ and $\Gamma$ are same C-type.

Conversely if $\Gamma$ is separable at label $k$, then the chart $\Gamma$ is clearly zero at label $k$. Thus we have done.

Lemma 11.([10, Lemma 6.1, Proposition 6.6 and Proposition 6.11]) Let $\Gamma$ be a minimal chart of type $\left(n_{1}, n_{2}, \cdots, n_{p}\right)$. Then we have the following:

(a) $n_{1}>1$ and $n_{p}>1$.

(b) If $n_{1}=2\left(\right.$ resp. $\left.n_{p}=2\right)$, then $n_{2}>1$ (resp. $\left.n_{p-1}>1\right)$.

(c) If $n_{1}=3$ (resp. $\left.n_{p}=3\right)$, then $n_{2}>1$ (resp. $\left.n_{p-1}>1\right)$.

Proof of Corollary 2. Let $\Gamma$ be a minimal chart with $w(\Gamma)=8$. Suppose that $\Gamma$ is zero at label $k$. Then by Theorem 1 there exists a chart $\Gamma^{\prime}$ with the same C-type of $\Gamma$ which is separable at label $k$. Here $w\left(\Gamma^{\prime}\right)=w(\Gamma)$. Hence there exist two subcharts $\Gamma^{*}, \Gamma^{* *}$ such that

(1) $\Gamma^{\prime}$ is the product of $\Gamma^{*}$ and $\Gamma^{* *}$,

(2) $w\left(\Gamma^{*}\right) \neq 0$ and $w\left(\Gamma^{* *}\right) \neq 0$.

Then we have

(3) $w\left(\Gamma^{*}\right)+w\left(\Gamma^{* *}\right)=w\left(\Gamma^{\prime}\right)=8$.

If either $\Gamma^{*}$ or $\Gamma^{* *}$ is not minimal, then $\Gamma^{\prime}$ is $\mathrm{C}$-move equivalent to a chart $\Gamma^{\prime \prime}$ with $w\left(\Gamma^{\prime \prime}\right)<w\left(\Gamma^{\prime}\right)=w(\Gamma)$. Namely the chart $\Gamma$ is $\mathrm{C}$-move equivalent to $\Gamma^{\prime \prime}$. This contradicts the fact that $\Gamma$ is minimal. Hence the two charts $\Gamma^{*}$ and $\Gamma^{* *}$ are minimal. Since there does not exist a minimal chart with at most three white vertices, we have $w\left(\Gamma^{*}\right) \geq 4$ and $w\left(\Gamma^{* *}\right) \geq 4$. Thus by (3), we have $w\left(\Gamma^{*}\right)=4$ and $w\left(\Gamma^{* *}\right)=4$. By Lemma 11(a) and (b), each of $\Gamma^{*}$ and $\Gamma^{* *}$ is of type $(4)$ or $(2,2)$. Therefore we complete the proof of Corollary 2.

Proof of Corollary 3. Let $\Gamma$ be a minimal $n$-chart with $w(\Gamma)=8$ of type $\left(n_{1}, n_{2}, \cdots, n_{p}\right)$ such that $\Gamma$ is not zero at any label. Then

(1) $n_{1}+n_{2}+\cdots+n_{p}=w(\Gamma)=8$,

(2) $n_{i} \geq 1$ for each $i(1 \leq i \leq p)$. 
By Lemma 11(a), we have $n_{1} \geq 2$ and $n_{p} \geq 2$. If necessary we change all the edges of label $k$ to ones of label $n-k$ for each $k=1,2, \cdots, n-1$ simultaneously, we can assume that

(3) $n_{1} \geq n_{p} \geq 2$.

There are four cases: (i) $p=1$ or 2 , (ii) $p=3$, (iii) $p=4$, (iv) $p \geq 5$.

Case (i). If $p=1$, then $\Gamma$ is of type (8). If $p=2$, then by (1) and (3) we have that the chart $\Gamma$ is of type $(6,2),(5,3)$ or $(4,4)$.

Case (ii). Suppose $p=3$. If $n_{3}=2$ or 3 , then $n_{2}=n_{p-1} \geq 2$ by Lemma 11(b) and (c). Thus by (1) and (3) we have that the chart $\Gamma$ is of type $(4,2,2),(3,3,2)$, $(2,4,2)$ or $(3,2,3)$.

If $n_{3} \geq 4$, then by (2) and (3) we have that $w(\Gamma)=n_{1}+n_{2}+n_{3} \geq 4+1+4=9$. This contradicts the fact $w(\Gamma)=8$.

Case (iii). Suppose $p=4$. If $n_{4}=2$ or 3 , then $n_{3}=n_{p-1} \geq 2$ by Lemma 11(b) and (c). Thus by (1), (2) and (3) we have that the chart $\Gamma$ is of type $(2,2,2,2)$, $(2,1,3,2)$ or $(3,1,2,2)$. However if $n_{1}=2$ or 3 , then $n_{2} \geq 2$ by Lemma 11 (b) and (c). Hence $\Gamma$ is of type $(2,2,2,2)$.

If $n_{4} \geq 4$, then by (2) and (3) we have that $w(\Gamma)=n_{1}+n_{2}+n_{3}+n_{4} \geq$ $4+1+1+4=10$. This contradicts the fact $w(\Gamma)=8$.

Case (iv). Suppose $p \geq 5$. There are two cases: (iv-1) $n_{p}=2$ or 3 , (iv-2) $n_{p} \geq 4$. Cases (iv-1). We have $n_{p-1} \geq 2$ by Lemma 11(b) and (c). Thus by (2), (3) we have that $w(\Gamma)=n_{1}+n_{2}+\cdots+n_{p-1}+n_{p} \geq n_{1}+n_{2}+n_{3}+n_{p-1}+n_{p} \geq$ $n_{1}+n_{2}+1+2+2=n_{1}+n_{2}+5$. Hence

$$
w(\Gamma) \geq n_{1}+n_{2}+5 .
$$

If $n_{1}=2$, then $n_{2} \geq 2$ by Lemma $11(\mathrm{~b})$. Thus $w(\Gamma) \geq 2+2+5=9$. This contradicts the fact $w(\Gamma)=9$.

If $n_{1} \geq 3$, then by $(2)$ we have $w(\Gamma) \geq 3+1+5=9$. This contradicts the fact $w(\Gamma)=9$.

Cases (iv-2). Since $n_{p} \geq 4$, by (2) and (3) we have that $w(\Gamma)=n_{1}+n_{2}+\cdots+$ $n_{p-1}+n_{p} \geq n_{1}+n_{2}+n_{3}+n_{p-1}+n_{p} \geq 4+1+1+1+4=11$. This contradicts the fact $w(\Gamma)=9$.

A chart $\Gamma$ belongs to the first class provided that

(i) $w(\Gamma)$ is odd, and

(ii) there does not exist a minimal chart $\Gamma^{\prime}$ such that $w\left(\Gamma^{\prime}\right)$ is odd and less than $w(\Gamma)$.

Corollary 12. If a minimal chart belongs to the first class, then the chart is not zero at any label. Namely if the type of the chart is $\left(m ; n_{1}, n_{2}, \cdots, n_{p}\right)$, then for each $i=1,2, \cdots, p$, we have $n_{i} \neq 0$.

Proof. Let $\Gamma$ be a minimal chart belonging to the first class. Suppose that the chart is zero at label $k$. Then by Theorem 1 there exists a chart $\Gamma^{\prime}$ with the same C-type of $\Gamma$ which is separable at label $k$. Thus there exist subcharts $\Gamma^{*}$ and $\Gamma^{* *}$ such that 
(1) $\Gamma^{\prime}$ is the product of the two charts $\Gamma^{*}$ and $\Gamma^{* *}$,

(2) $w\left(\Gamma^{*}\right) \neq 0$ and $w\left(\Gamma^{* *}\right) \neq 0$.

Then we have

(3) $w\left(\Gamma^{*}\right)+w\left(\Gamma^{* *}\right)=w\left(\Gamma^{\prime}\right)$.

Since $\Gamma^{\prime}$ and $\Gamma$ are same C-type, we have $w\left(\Gamma^{\prime}\right)=w(\Gamma)$. Since $\Gamma$ belongs to the first class, we have that $w\left(\Gamma^{\prime}\right)(=w(\Gamma))$ is odd. Thus $w\left(\Gamma^{*}\right)$ or $w\left(\Gamma^{* *}\right)$ is odd less than $w(\Gamma)$. Since $\Gamma$ belongs to the first class, either $\Gamma^{*}$ or $\Gamma^{* *}$ is not a minimal chart. Hence $\Gamma^{\prime}$ is C-move equivalent to a chart $\Gamma^{\prime \prime}$ with $w\left(\Gamma^{\prime \prime}\right)<w\left(\Gamma^{\prime}\right)=w(\Gamma)$. Namely the chart $\Gamma$ is $\mathrm{C}$-move equivalent to $\Gamma^{\prime \prime}$. This contradicts the fact that $\Gamma$ is minimal. Therefore $\Gamma$ is not zero at any label.

Proof of Corollary 4. There does not exist a minimal chart $\Gamma$ with $w(\Gamma)=1,2$, or 3 . Further there does not exist a minimal chart $\Gamma$ with $w(\Gamma)=5([16])$. Furthermore there does not exist a minimal chart $\Gamma$ with $w(\Gamma)=7$ ([9], [10], [11], [12], [13]). Hence any chart with nine white vertices belongs to the first class. Thus any minimal chart with nine white vertices is not zero at any label by Corollary 12 .

Let $\Gamma$ be a minimal chart with $w(\Gamma)=11$. Suppose that $\Gamma$ is zero at label $k$. Then by Theorem 1 there exists a chart $\Gamma^{\prime}$ with the same C-type of $\Gamma$ which is separable at label $k$. Here $w\left(\Gamma^{\prime}\right)=w(\Gamma)$. Hence there exist two subcharts $\Gamma^{*}, \Gamma^{* *}$ such that

(1) $\Gamma^{\prime}$ is the product of the two charts $\Gamma^{*}$ and $\Gamma^{* *}$,

(2) $w\left(\Gamma^{*}\right) \neq 0$ and $w\left(\Gamma^{* *}\right) \neq 0$.

Then we have

(3) $w\left(\Gamma^{*}\right)+w\left(\Gamma^{* *}\right)=w\left(\Gamma^{\prime}\right)=11$.

There are five cases:

(i) one of $w\left(\Gamma^{*}\right)$ and $w\left(\Gamma^{* *}\right)$ equals 1 and the other equals 10 ,

(ii) one of $w\left(\Gamma^{*}\right)$ and $w\left(\Gamma^{* *}\right)$ equals 2 and the other equals 9 ,

(iii) one of $w\left(\Gamma^{*}\right)$ and $w\left(\Gamma^{* *}\right)$ equals 3 and the other equals 8 ,

(iv) one of $w\left(\Gamma^{*}\right)$ and $w\left(\Gamma^{* *}\right)$ equals 4 and the other equals 7 , and

(v) one of $w\left(\Gamma^{*}\right)$ and $w\left(\Gamma^{* *}\right)$ equals 5 the other equals 6 .

But in any case, either $\Gamma^{*}$ or $\Gamma^{* *}$ is not minimal. Hence $\Gamma^{\prime}$ is C-move equivalent to a chart $\Gamma^{\prime \prime}$ with $w\left(\Gamma^{\prime \prime}\right)<w\left(\Gamma^{\prime}\right)=w(\Gamma)$. Namely the chart $\Gamma$ is $\mathrm{C}$-move equivalent to $\Gamma^{\prime \prime}$. This contradicts the fact that $\Gamma$ is minimal. Therefore any minimal chart $\Gamma$ with $w(\Gamma)=11$ is not zero at any label.

Proof of Corollary 5. Let $\Gamma$ be a minimal $n$-chart with $w(\Gamma)=9$ of type $\left(n_{1}, n_{2}, \cdots, n_{p}\right)$. Then 
(1) $n_{1}+n_{2}+\cdots+n_{p}=w(\Gamma)=9$.

By Corollary 4, we have

(2) $n_{i} \geq 1$ for each $i(1 \leq i \leq p)$.

By Lemma 11(a), we have $n_{1} \geq 2$ and $n_{p} \geq 2$. If necessary we change all the edges of label $k$ to ones of label $n-k$ for each $k=1,2, \cdots, n-1$ simultaneously, we can assume that

(3) $n_{1} \geq n_{p} \geq 2$, and

(4) if $n_{1}=n_{p}$ and $p \geq 4$, then $n_{2} \geq n_{p-1}$.

If $p=1$, then $\Gamma$ is of type (9). If $p=2$, then by (1) and (3) the chart $\Gamma$ is of type $(7,2),(6,3)$ or $(5,4)$.

Suppose $p \geq 3$. There are two cases: (i) $n_{p}=2$ or 3 , (ii) $n_{p} \geq 4$.

Case (i). By Lemma 11(b) and (c), we have $n_{p-1} \geq 2$. There are four cases: (i-1) $p=3$, (i-2) $p=4,(\mathrm{i}-3) p=5,(\mathrm{i}-4) p \geq 6$.

Case (i-1). Suppose $p=3$. By (1), (3) and $n_{2}=n_{p-1} \geq 2$, the chart $\Gamma$ is of type $(5,2,2),(4,3,2),(3,4,2),(2,5,2),(4,2,3)$ or $(3,3,3)$.

Case (i-2). Suppose $p=4$. If $n_{1}=2$ or 3 , then $n_{2} \geq 2$ by Lemma $11(\mathrm{~b})$ and (c). Thus by (1), (3), (4) and $n_{3}=n_{p-1} \geq 2$, the chart $\Gamma$ is of type $(2,3,2,2)$ or $(3,2,2,2)$.

If $n_{1}=4$, by (1), (2), (3) and $n_{3}=n_{p-1} \geq 2$, then $\Gamma$ is of type $(4,1,2,2)$

If $n_{1} \geq 5$, then by (2), (3) and $n_{3}=n_{p-1} \geq 2$, we have $w(\Gamma)=n_{1}+n_{2}+n_{3}+n_{4} \geq$ $5+1+2+2=10$. This contradicts the fact $w(\Gamma)=9$.

Case (i-3). Suppose $p=5$. If $n_{1}=2$, then $n_{2} \geq 2$ by Lemma 11(b). Thus by (1), (2), (3) and $n_{4}=n_{p-1} \geq 2$, we have that the chart $\Gamma$ is of type $(2,2,1,2,2)$.

If $n_{1}=3$, then $n_{2} \geq 2$ by Lemma 11(c). Thus by (2), (3) and $n_{4}=n_{p-1} \geq 2$ we have $w(\Gamma)=n_{1}+n_{2}+n_{3}+n_{4}+n_{5} \geq 3+2+1+2+2=10$. This contradicts the fact $w(\Gamma)=9$.

If $n_{1} \geq 4$, then by (2), (3) and $n_{4}=n_{p-1} \geq 2$ we have $w(\Gamma)=n_{1}+n_{2}+n_{3}+$ $n_{4}+n_{5} \geq 4+1+1+2+2=10$. This contradicts the fact $w(\Gamma)=9$.

Case (i-4). Suppose $p \geq 6$. If $n_{1}=2$ or 3 , then $n_{2} \geq 2$ by Lemma 11(b) and (c). Thus by (2), (3) and $n_{p-1} \geq 2$, we have $w(\Gamma)=n_{1}+n_{2}+\cdots+n_{p-1}+n_{p} \geq$ $n_{1}+n_{2}+n_{3}+n_{4}+n_{p-1}+n_{p} \geq 2+2+1+1+2+2=10$. This contradicts the fact $w(\Gamma)=9$.

If $n_{1} \geq 4$, then by (2), (3) and $n_{p-1} \geq 2$ we have $w(\Gamma)=n_{1}+n_{2}+\cdots+n_{p-1}+$ $n_{p} \geq n_{1}+n_{2}+n_{3}+n_{4}+n_{p-1}+n_{p} \geq 4+1+1+1+2+2=11$. This contradicts the fact $w(\Gamma)=9$.

Case (ii). If $p=3$, then by (1), (2) and (3) we have $\Gamma$ is of type $(4,1,4)$.

If $p \geq 4$, then by (2) and (3) we have $w(\Gamma)=n_{1}+n_{2}+\cdots+n_{p-1}+n_{p} \geq$ $n_{1}+n_{2}+n_{3}+n_{p} \geq 4+1+1+4=10$. This contradicts the fact $w(\Gamma)=9$. 


\section{References}

[1] J. S. Carter and M. Saito, Knotted surfaces and their diagrams, Mathematical Surveys and Monographs, 55, American Mathematical Society, Providence, RI, (1998).

[2] J. S. Carter, S. Kamada, and M. Saito, Alexander numbering of knotted surface diagrams,Proc. Amer. Math. Soc., 128(2000), 3761-3771.

[3] I. Hasegawa, The lower bound of the w-indices of non-ribbon surface-links, Osaka J. Math., 41(2004), 891-909.

[4] S. Ishida, T. Nagase and A. Shima, Minimal n-charts with four white vertices, J. Knot Theory Ramifications, 20(2011), 689-711.

[5] S. Kamada, Surfaces in $R^{4}$ of braid index three are ribbon, J. Knot Theory Ramifications, 1(2)(1992), 137-160.

[6] S. Kamada, An observation of surface braids via chart description, J. Knot Theory Ramifications, 5(4)(1996), 517-529.

[7] S. Kamada, Braid and Knot Theory in Dimension Four, Mathematical Surveys and Monographs, 95, American Mathematical Society, (2002).

[8] T. Nagase, D. Nemoto and A. Shima, There exists no minimal n-chart of type $(2,2,2)$, Proc. Sch. Sci. Tokai Univ., 46(2011), 1-31.

[9] T. Nagase and A. Shima, Properties of minimal charts and their applications I, J. Math. Sci. Univ. Tokyo, 14(2007), 69-97.

[10] T. Nagase and A. Shima, Properties of minimal charts and their applications II, Hiroshima Math. J., 39(2009), 1-35.

[11] T. Nagase and A. Shima, Properties of minimal charts and their applications III, Tokyo J. Math., 33(2010), 373-392.

[12] T. Nagase and A. Shima, Properties of minimal charts and their applications IV: Loops, to appear J. Math. Sci. Tokyo J. Math. (arXiv:1603.04639).

[13] T. Nagase and A. Shima, Properties of minimal charts and their applications $V$-, in preparation.

[14] T. Nagase and A. Shima, Gambits in charts, J. Knot Theory Ramifications, 24(9) (2015), 1550052 (21 pages).

[15] T. Nagase, A. Shima and H. Tsuji, The closures of surface braids obtained from minimal $n$-charts with four white vertices, J. Knot Theory Ramifications, 22(2)(2013) 1350007 (27 pages).

[16] M. Ochiai, T. Nagase and A. Shima, There exists no minimal n-chart with five white vertices, Proc. Sch. Sci. Tokai Univ., 40(2005), 1-18.

[17] K. Tanaka, A Note on CI-moves, Intelligence of Low Dimensional Topology 2006 Eds. J. Scott Carter et al. (2006), 307-314. 